\title{
The Design of Seismic Migration Complex-Valued Finite Impulse Response Filters
}

\author{
Wail A. Mousa \\ Department of Electrical Engineering \\ King Fahd University of Petroleum \& Minerals, \\ Dhahran 31261, Saudi Arabia \\ Email: wailmousa@kfupm.edu.sa
}

\begin{abstract}
This paper proposes a novel way to design seismic migration Finite Impulse Response (FIR) digital filters using the Newton minimization algorithm. The algorithm requires computing the inverse of the Jacobian matrix, which is nonsquare for the seismic migration filters problem. In this case, we suggest using the Moore-Penrose pseudo-inverse to obtain the inverse of the Jacobian matrix. The proposed design algorithm running time is about 8 times faster than the recently proposed $L_{1}$-norm algorithm. Furthermore, the proposed method results in seismic migration filters that lead to practically stable seismic images.
\end{abstract}

\section{INTRODUCTION}

Seismic data analysis is performed for numerous applications such as petroleum exploration, determination of the earth's core structure, monitoring earthquakes, etc., [1]. The oil industry is interested in imaging the earth subsurface layers using seismic waveforms. Due to factors related to the geological structure of the earth itself, the layers appearing in seismic sections are incorrectly positioned and the resolution of such sections are also affected. Hence, seismic imaging is very critical in obtaining accurate images of the subsurface [1].

There exist many proposed techniques to image the recorded seismic data [1]-[4]. Seismic migration (using as frequencyspace $(f-x))$ filters have been developed to address strong lateral variations of the subsurface structure velocities, a common occurrence in the earth subsurface layers [1], [5]-[8]. If two-dimensional (2-D) seismic data is considered, the $f-x$ seismic imaging process requires filtering seismic wavefields in the $f-x$ domain. The filtering is based on the following (normalized) one-dimensional (1-D) desired wavenumber response [8]:

$$
H_{d}\left(k_{x}\right)=\exp \left(j b \sqrt{k_{c}^{2}-k_{x}^{2}}\right),
$$

where $k_{x}$ is the normalized horizontal wavenumber. Note that $b=\Delta z / \Delta x$ is a constant, where $\Delta z$ is the depth-sampling interval and $\Delta x$ is the spatial sampling interval. Note that $k_{c}$ is the normalized cut-off wavenumber. In the case of acoustic seismic imaging it can be given by:

$$
k_{c}=\frac{\omega}{c},
$$

where $\omega$ is the angular frequency and $c$ is the velocity of the medium. Both the magnitude and the phase spectra are of even symmetry and, hence, the filter coefficients are complex-valued.

To accommodate for the strong lateral variations, researchers have, over the last two decades, focused on approximating Eq. (1) by an $N$-length ( $N$ is odd) non-causal Finite Impulse Response (FIR) digital filter that is of even symmetry (see for example [6], [7], [9]-[13]). This filter can be mathematically represented as:

$$
H\left(e^{j k_{x}}\right)=\sum_{n=0}^{M}(2-\delta[n]) h[n] \cos \left(n k_{x}\right),
$$

where $h[n]$ represents the seismic migration FIR filter coefficients, which are complex-valued, $M=\frac{N-1}{2}$, and $\delta[n]$ is the unit sample sequence.

In this paper, we show that the seismic migration FIR filter design problem can be formulated and solved using the Newton's method. The resulting filters are efficient than some existing techniques such as the $L_{1}$-norm designed seismic migration filters reported in [8] and result in practically stable seismic images. We present the Newton's-based seismic migration FIR filters design method and algorithm in section 2. Next, in section 3, we present simulation results and comparisons of designed seismic migration FIR filters. In section 4, we discuss a few practical algorithm points. Finally, we conclude the paper in section 5 .

\section{The Newton's-BASed Seismic Migration FIR FILTERS}

The aim is to determine the set of the seismic migration FIR filter coefficients $h[n]$ such that $H\left(e^{j k_{x}}\right)$ given by Eq. 3 fits the ideal (desired) wavenumber response $H_{d}\left(k_{x}\right)$ given by Eq. 1. When sampling the wavenumber independent variable $k_{x}$ into $R$ samples, we can restate the seismic migration FIR filter design problem (based on [8]) as follows:

$$
\mathbf{H}_{d}=\mathbf{C h}
$$

or, equivalently,

$$
\mathbf{F}(\mathbf{h})=\mathbf{H}_{d}-\mathbf{C h}=\mathbf{0},
$$


where

$$
\begin{gathered}
\mathbf{h}=\left[\begin{array}{lllll}
h[0] & h[1] & \ldots & h[M]
\end{array}\right]^{*} \\
\mathbf{C}=\left[\begin{array}{ccccc}
1 & \cos \left(k_{x_{1}}\right) & \cos \left(2 k_{x_{1}}\right) & \ldots & \cos \left(M k_{x_{1}}\right) \\
1 & \cos \left(k_{x_{2}}\right) & \cos \left(2 k_{x_{2}}\right) & \ldots & \cos \left(M k_{x_{2}}\right) \\
\vdots & \vdots & \vdots & \ldots & \vdots \\
1 & \cos \left(k_{x_{R}}\right) & \cos \left(2 k_{x_{R}}\right) & \ldots & \cos \left(M k_{x_{R}}\right)
\end{array}\right]
\end{gathered}
$$

and, finally,

$$
\mathbf{H}=\left[\begin{array}{llll}
H_{d}\left(k_{x_{1}}\right) & H_{d}\left(k_{x_{2}}\right) & \ldots & H_{d}\left(k_{x_{R}}\right)
\end{array}\right]^{*} .
$$

Note that, in this case, $\mathbf{H}_{d}$ is of dimension $R \times 1$, C is of dimension $R \times M$ and $\mathbf{h}$ is a vector of size $M \times 1$. Also, note that $*$ denotes the Hermitian conjugate.

Using the first a few terms of Taylor series of $\mathbf{F}(\mathbf{h})=\mathbf{0}$ (we showed in Eq. 5) in the vicinity of a possible seismic migration FIR filter solution $\mathbf{h}$, the set of the seismic migration filter coefficients that satisfies Eq. 5 can iteratively be found using the Newton's method [14]. That is:

$$
\mathbf{h}^{k+1}=\mathbf{h}^{k}-\left[\mathbf{B}\left(\mathbf{h}^{k}\right)\right]^{-1} \mathbf{F}\left(\mathbf{h}^{k}\right),
$$

where $\mathbf{B}$ is the Jacobian matrix and is given by:

$$
\mathbf{B}=\left[\begin{array}{cccc}
\frac{\partial F_{1}}{\partial h[0]} & \frac{\partial F_{1}}{\partial h[1]} & \cdots & \frac{\partial F_{1}}{\partial h[M-1]} \\
\frac{\partial F_{2}}{\partial h[0]} & \frac{\partial F_{2}}{\partial h[1]} & \cdots & \frac{\partial F_{2}}{\partial h[M-1]} \\
\vdots & \vdots & \ddots & \vdots \\
\frac{\partial F_{R}}{\partial h[0]} & \frac{\partial F_{R}}{\partial h[1]} & \cdots & \frac{\partial F_{R}}{\partial h[M-1]}
\end{array}\right] .
$$

Note that $F_{1}, F_{2}, \ldots, F_{R}$ represent components of Eq. 5 . We can easily show that $\mathbf{B}=\mathbf{C}$, which means that the Jacobian matrix is independent of $\mathbf{h}$. Hence, we can write Eq. 9 as:

$$
\mathbf{h}^{k+1}=\mathbf{h}^{k}-\mathbf{C}^{-1} \mathbf{F}\left(\mathbf{h}^{k}\right) .
$$

Note that $\mathbf{C}$ is not invertible since it is of dimensions $R \times M$, where usually $R \gg M$. Hence, we suggest using the MoorePenrose pseudo-inverse [15] of the Jacobian matrix $\mathbf{C}$ instead.

\section{A. Proposed Newton-based Design Algorithm for Seismic Mi- gration FIR Filters}

The proposed Newton's algorithm for designing the seismic migration FIR filters is, hence, summarized as follows. The algorithm starts with an arbitrary complex-valued vector $\mathbf{h}^{0}$. Choosing a filter length $N$ and a wavenumber cut-off $k_{c}$, then for the $k^{t h}$ iteration, we will use the following steps:

1) Formulate the matrices $\mathbf{C}$ and $\mathbf{H}_{d}$ based on Eqs. 7 and 8 , respectively.

2) Compute $\mathbf{F}\left(\mathbf{h}^{k}\right)$ using Eq. 5.

3) Compute the Moore-Penrose pseudo-inverse of the Jacobian matrix $\mathbf{C}$.

4) Obtain $\mathbf{h}^{k+1}$ using Eq. 11.

If the mean square error between $\mathbf{h}^{k}$ and $\mathbf{h}^{k+1}$ is less than or equal to a predefined threshold $\epsilon$, stop the algorithm; otherwise, repeat steps $2-4$.
Table I

NUMBER OF ITERATIONS AND RUNNING DESIGN TIME FOR THE DESIGNED SEISMIC MIGRATION FIR FILTERS SHOWN IN FIGURE 1 BASED ON THE PROPOSED NEWTON'S ALGORITHM AND THE $L_{1}$-NORM DESIGN ALGORITHM FOR $N=25$.

\begin{tabular}{|c|c|c|}
\hline Design method & No. of iterations & Time, $\mathrm{s}$ \\
\hline \hline Newton $\left(\mathrm{MSE}=10^{-35}\right.$ ) & 49 & 0.014728 \\
\hline$L_{1}$-norm & 31 & 0.120061 \\
\hline
\end{tabular}

\section{Simulation Results}

In this section, a 1-D complex-valued FIR seismic migration digital filter is designed using the Newton algorithm given in section 2. Figure 1 show the Newton's seismic migration FIR filter wavenumber magnitude and phase responses, respectively, for a normalized $k_{c}=0.25, \Delta z=2 \mathrm{~m}, \Delta x=10$ $\mathrm{m}, N=25$ with a MSE of $\epsilon=10^{-35}$, and $R=512$. Clearly the response of the designed filters approximate the response of the desired filters and, hence, can result in stable seismic migration [7]. Also, from Figures 1 (a) and (b), we see that the proposed method results in a better magnitude response as well as comparable phase response with that of the $L_{1}$-norm seismic migration FIR filter reported in [8]. In addition, Figure 1 (c) plots the mean-square error between consecutive iterations versus the number of iterations required to meet the pre-defined stopping threshold for the designed filter. The algorithms required 49 iterations to converge with $\epsilon=10^{-35}$. Also, Table I shows that the running time for designing the seismic migration FIR filters using the proposed method is about 8 times faster than the one designed based on the $L_{1}$-norm algorithm. This is despite the fact that it took more iterations than the $L_{1}$-norm algorithm.

Finally, to prove the concept, we designed and stored in a look-up table (e.g., [9], [16]) a set of 1260 frequency-velocity dependent seismic migration FIR filters with a maximum frequency $f_{\max }=45 \mathrm{~Hz}$, a filter length of $N=25$ coefficients, and the MSE $\epsilon=10^{-35}$. On account of symmetry conditions, only 13 out of the 25 coefficients were stored. Also, the used number of wavenumber points $R=512$. The filters were implemented in a poststack explicit depth extrapolation method as reported by Holberg [9]. In this method, the filter coefficients are implemented as a spatially varying convolution. In this case, lateral velocity variations can be accommodated for data similar to the SEG/EAGE salt model (see [7], [8]). The SEG/EAGE salt data parameters were $\Delta z=2 \mathrm{~m}, \Delta x=10 \mathrm{~m}$. Figure 2 shows the imaging result obtained by using the 25 -length seismic migration filter coefficients designed with the proposed Newton's algorithm. Furthermore, Figure 2 shows that the designed Newton-based filters lead to practically stable seismic images. That is, when using such designed filters (meeting the seismic migration design requirements) in such an extrapolation method, the seismic data amplitude values from the pervious extrapolation step are neither amplified nor attenuated. Hence, the images are practically stable. 


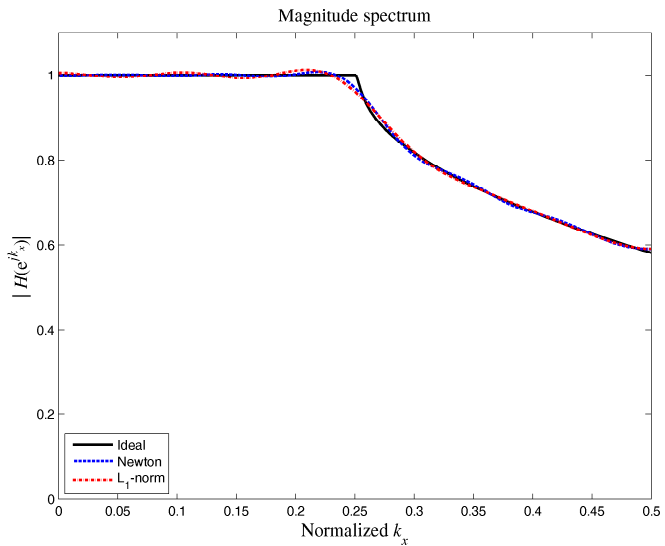

(a)

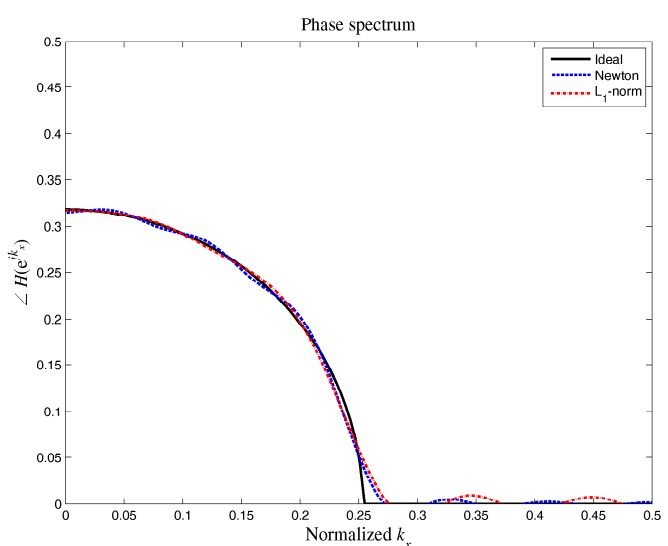

(b)

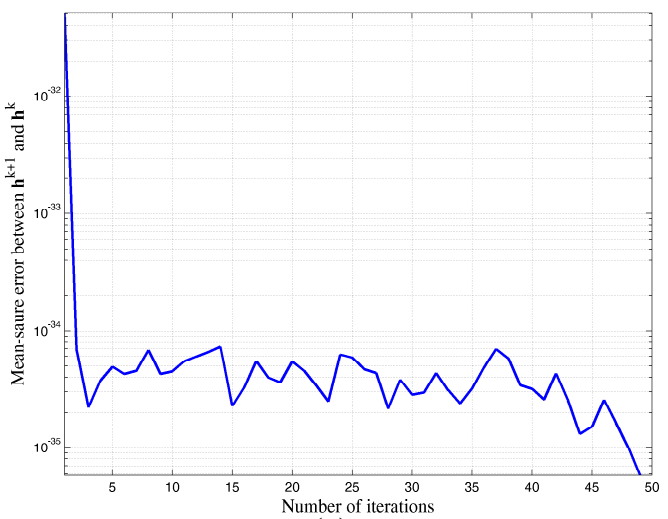

(c)

Figure 1. Accuracy of the Newton-based designed seismic migration FIR filters (also compared with ones designed using the $L_{1}$-norm minimization algorithm) with a normalized $k_{c}=0.25, N=25$ and a MSE of $\epsilon=10^{-35}$. (a) and (b) display their magnitude and phase responses within the passband compared with the desired wavenumber response provided in Eq. (1). (c) show the MSE between $\mathbf{h}^{k+1}$ and $\mathbf{h}^{k}$ versus the number of iterations for the designed 1-D seismic migration filter.

\section{Discussion}

The initialization of the algorithm when designing 1-D seismic migration FIR filters using the proposed Newton-based method is important to discuss. A good choice could be to start with an approximation of the ideal filter response to

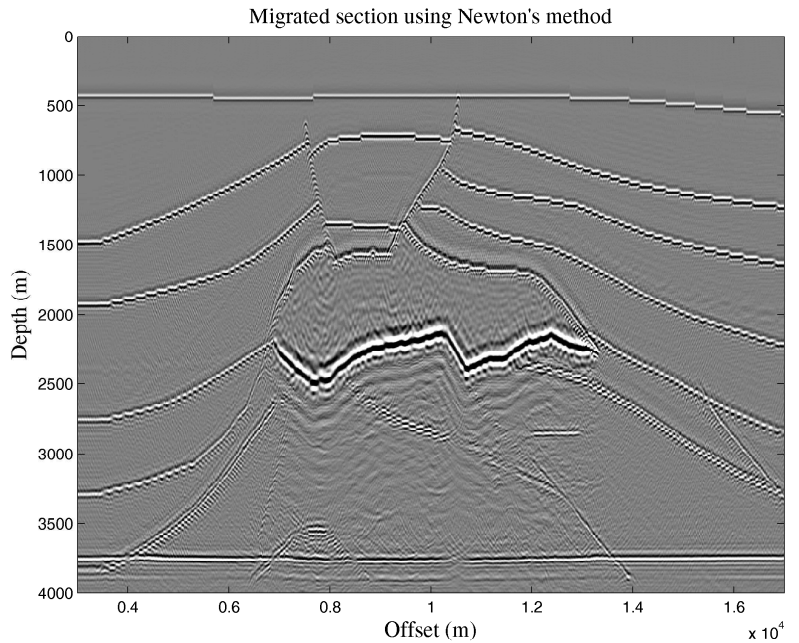

Figure 2. To prove the concept, image of the seismic SEG/EAGE salt data obtained using the proposed Newton-based algorithm with the seismic migration FIR filters $(N=25)$.

initialize the algorithm. This is obtained by inverse Fourier transforming the ideal filter wavenumber response into the space domain, saving a number of the early iterations. Another way, which we have adopted here, is to make $\mathbf{h}^{0}$ equal to the least square solution (see [8]). Starting with any of the above suggestions will decrease the probability of the proposed algorithm diverging. This would further be investigated in future work.

\section{CONCLUSIONS}

In this paper, we demonstrated that the problem of designing seismic migration FIR filters can be formulated and solved based on the Newton's method. The proposed design algorithm running time is about 8 times faster than the recently proposed $L_{1}$-norm algorithm. We showed an application of the filters on the SEG/EAGE benchmark seismic model data set. The designed Newton-based filters lead to practically stable seismic images. Finally, it is straight forward to extend the paper's proposed methods to design efficient 2-D seismic migration FIR filters, that could used to migrate 3 -D seismic data. This is an undergoing research work.

\section{ACKNOWLEDGMENTS}

The author thanks King Fahd University of Petroleum \& Minerals (KFUPM) for sponsoring this work.

\section{REFERENCES}

[1] O. Yilmaz, Ed., Seismic Data Analysis: Processing, Inversion, and Interpretation of Seismic Data, 2nd ed. Society of Exploration Geophysicists, 2001.

[2] A. Duchkov, F. Andersson, and M. de Hoop, "Discrete almost-symmetric wave packets and multiscale geometrical representation of (seismic) waves," IEEE Transactions on Geoscience and Remote Sensing, vol. 48, no. 9 , pp. $3408-3423$, sept. 2010.

[3] R. Streich and J. V. D. Kruk, "Accurate imaging of multicomponent gpr data based on exact radiation patterns," IEEE Transactions on Geoscience and Remote Sensing, vol. 45, no. 1, pp. 93 -103, jan. 2007. 
[4] T. Sakamoto, S. Kidera, and T. Sato, "Seabed algorithm and comments on ldquo;modeling and migration of 2-d georadar data: A stationary phase approach rdquo;", IEEE Transactions on Geoscience and Remote Sensing, vol. 45, no. 10, p. 3300, oct. 2007.

[5] J. T. Etgen, "Stability of explicit depth extrapolation through laterallyvarying media," SEG 1994 Expanded Abstracts, 1994

[6] J. W. Thorbecke, K. Wapenaar, and G. Swinnen, "Design of one-way wavefield extrapolation operators, using smooth functions in WLSQ optimization," Geophysics, vol. 69, no. 4, pp. 1037-1045, 2004.

[7] W. A. Mousa, M. V. D. Baan, S. Boussakta, and D. McLernon, "Designing stable operators for explicit depth extrapolation of 2-D \& 3-D wavefields using projections onto convex sets," Geophysics, vol. 74, no. 2, pp. P.S33-S45, March-April 2009.

[8] W. A. Mousa, "Imaging of the SEG/EAGE salt model seismic data using sparse $f-x$ finite-impulse-response wavefield extrapolation filters," IEEE Transactions on Geoscience and Remote Sensing, vol. 52, no. 5, pp. 2700-2714, May 2014

[9] O. Holberg, "Towards optimum one-way wave propagation," Geophysical Prospecting, vol. 36, pp. 99-114, 1988.

[10] D. Hale, "Stable explicit depth extrapolation of seismic wavefields," Geophysics, vol. 56, pp. 1770 - 1777, 1991.

[11] L. J. Karam and J. H. McClellan, "Complex Chebyshev approximation for FIR filter design," IEEE Trans. on Circuits and Systems, vol. 42 no. 3, pp. 207 - 216, March 1995.

[12] R. Soubaras, "Explicit 3-D migration using equiripple polynomial expansion and Laplace synthesis," Geophysics, vol. 61, no. 5, pp. 13861393, 1996.

[13] M. M. Naseer and W. A. Mousa, "Linear complementarity problem: A novel approach to design finite-impulse response wavefield extrapolation filters," Geophysics, vol. 80, no. 2, pp. S55-S63, 2015

[14] T. Sauer, Numerical Analysis, 2nd ed. Pearson, 2011.

[15] E. H. Moore, "On the reciprocal of the general algebraic matrix," Bulletin of the American Mathematical Society, vol. 26, no. 9, p. 394395 , 1920.

[16] G. Blacquire, H. W. J. Debeye, C. P. A. Wapenaar, and A. J. Berkhout, "3D table-driven migration1," Geophysical Prospecting, vol. 37, no. 8 pp. 925-958, 1989. 